\title{
TRANSIENT COMPUTATIONAL FLUID DYNAMICS ANALYSIS OF EMERGENCY CORE COOLING INJECTION AT NATURAL CIRCULATION CONDITIONS
}

\author{
Martina Scheuerer and Johannes Weis \\ Gesellschaft für Anlagen- und Reaktorsicherheit, \\ Forschungsinstitute, 85748 Garching, Germany
}

\begin{abstract}
Within the framework of the European Nuclear Reactor Integrated Simulation Project (NURISP), Computational Fluid Dynamics (CFD) software is validated for the simulation of the thermohydraulics of pressurized thermal shocks. A proposed validation experiment is the test series performed within the OECD ROSA V project in the Large Scale Test Facility (LSTF). The LSTF is a 1:48 volume-scaled model of a four-loop Westinghouse pressurized water reactor (PWR). ROSA V Test T1-1 investigates temperature stratification under natural circulation conditions.
\end{abstract}

This paper describes calculations which were performed with the ANSYS CFD software for emergency core cooling injection into one loop at single-phase flow conditions. Following the OECD/NEA CFD Best Practice Guidelines (Mahaffy, 2007) the influence of grid resolution, discretisation schemes, and turbulence models (Shear Stress Transport and Reynolds Stress Model) on the mixing in the cold leg were investigated. A half-model was used for these simulations.

The transient calculations were started from a steady-state solution at natural circulation conditions. The final calculations were obtained in a complete model of the downcomer. The results are in good agreement with data.

\section{INTRODUCTION}

Within the framework of the European NURISP Project, CFD software is validated for the simulation of the thermo-hydraulics of pressurized thermal shocks (PTS). These phenomena are important for plant life extension and aging.

PTS phenomena are characterised by multi-dimensional non-equilibrium flow conditions. They occur when emergency core cooling (ECC) systems are actuated during loss-of-coolant accidents (LOCA) in pressurized water reactors (PWR). In these situations, cold water is injected into the cold legs where it mixes with the hot primary coolant, and flows then into the pressure vessel. Insufficient mixing may cause temperature stratification and steam condensation on the cold surface of the injected water. Flow oscillations may then cause thermal stresses in the surrounding structures and lead to a reduced vessel life time.

Because of their importance, PTS effects have been studied experimentally and numerically. The available investigations can be subdivided into separate-effect studies and combined-effect studies. The separate-effect studies deal only with a single aspect of PTS, like free surface flow or generic condensation (Scheuerer, 2002). The combined-effect studies are performed in realistic reactor configurations, and examine the interaction of all relevant effects. Because of their complexity and cost, only few data are available.

Combined-effect PTS data have been collected in the Upper Plenum Test Facility (UPTF). UPTF is a 1:1 model of a $1300 \mathrm{MWe}$ Siemens/KWU PWR. In the UPTF Test 1, ECC injection into stagnant hot water at small-break LOCA conditions was investigated. The CFD simulation by Willemsen and Komen (2005) 
showed that buoyancy production terms have to be included in the applied eddy turbulence models in order to achieve a good representation of the temperature stratification in the cold leg.

The influence of density differences on the mixing of the primary loop inventory and ECC water in a PWR was analyzed at the Rossendorf Coolant Mixing (ROCOM) test facility. ROCOM is a 1:5-scaled model of a German PWR. It is equipped with instrumentation, which delivers high-resolution information for concentration fields. Experimental and numerical investigations by Höhne et al. (2005) show that buoyancy dominates slug propagation. ECC water reaching the downcomer flows in an almost vertical path and reaches the lower downcomer sensor directly below the inlet nozzle.

The ROSA V experiments which were performed in the Large Scale Test Facility LSTF (JAERI, 2003) belong also to the combined-effect category. In the ROSA V experiments, the three-dimensional temperature field was measured in the cold leg and downcomer under realistic reactor conditions. The test series performed within the OECD ROSA V project was selected as validation case in the NURISP project because of its detailed temperature data. In this paper, CFD simulations and data for the ROSA V Test 1-1 are compared and assessed. As the experimental data is proprietory, absolute temperature values can not be provided.

\section{ROSA V EXPERIMENT}

The LSTF emulates a Westinghouse-type four-loop 3423 MW thermal power PWR by a full-height and $1 / 48$ volumetrically-scaled two-loop system (JAERI, 2008). The cold legs are identical, and consist of straight and elbow parts which are attached to the main coolant pumps, see Fig. 1. However, the ECC injection nozzles in the cold legs differ in shape and location. ECC Nozzle A is perpendicular to the main pipe simulating VVER reactor type conditions, while ECC Nozzle B forms a $45^{\circ}$ angle with the cold leg.

The ROSA V Test 1-1 was sponsored by the OECD program. It was performed in 2006. The goal of the experimental program was to investigate temperature stratification under natural circulation conditions, and to provide data for the validation of CFD software. To this end, temperatures were measured with thermocouple rakes in the cold legs below the injection nozzle (TE1), and at two cross-sectional planes between the injection nozzle and the downcomer (TE2, TE3). Three thermocouples were mounted vertically below the injection nozzle. The rakes in the cold leg consisted of 21 thermocouples in three columns and seven rows. Additionally, 18 thermocouples were installed in the downcomer below the cold legs (TE4), see Fig. 2.

Fig. 3 shows the 21-thermocouple installation representative of positions TE2 and TE3 in the cold leg. Fig. 4 shows how the 18-thermocouples are installed in the downcomer. The nominal accuracy of the thermocouple measurements is $\pm 2.75 \mathrm{~K}$. The photographs include the supports which were used to hold the thermocouple probes. These are relatively large. It can therefore be expected that they influence thermal mixing and temperature stratification in the cold legs, and lead to additional experimental uncertainty.

The ROSA V Test 1-1 experiment was performed in several steps. It started with separated ECC injections into Cold Leg A, followed by injection into Cold Leg B at $15.5 \mathrm{MPa}$, and at $100 \%$ primary inventory with a core power corresponding to $2 \%$ of the scaled nominal power. Thereafter the water level was reduced to $80 \%, 70 \%$ and $50 \%$ of the inventory, with 10-minute intervals for re-stabilisation. For every water level the ECC injection was performed in sequence, first into Cold Leg A, then into Cold Leg B. 


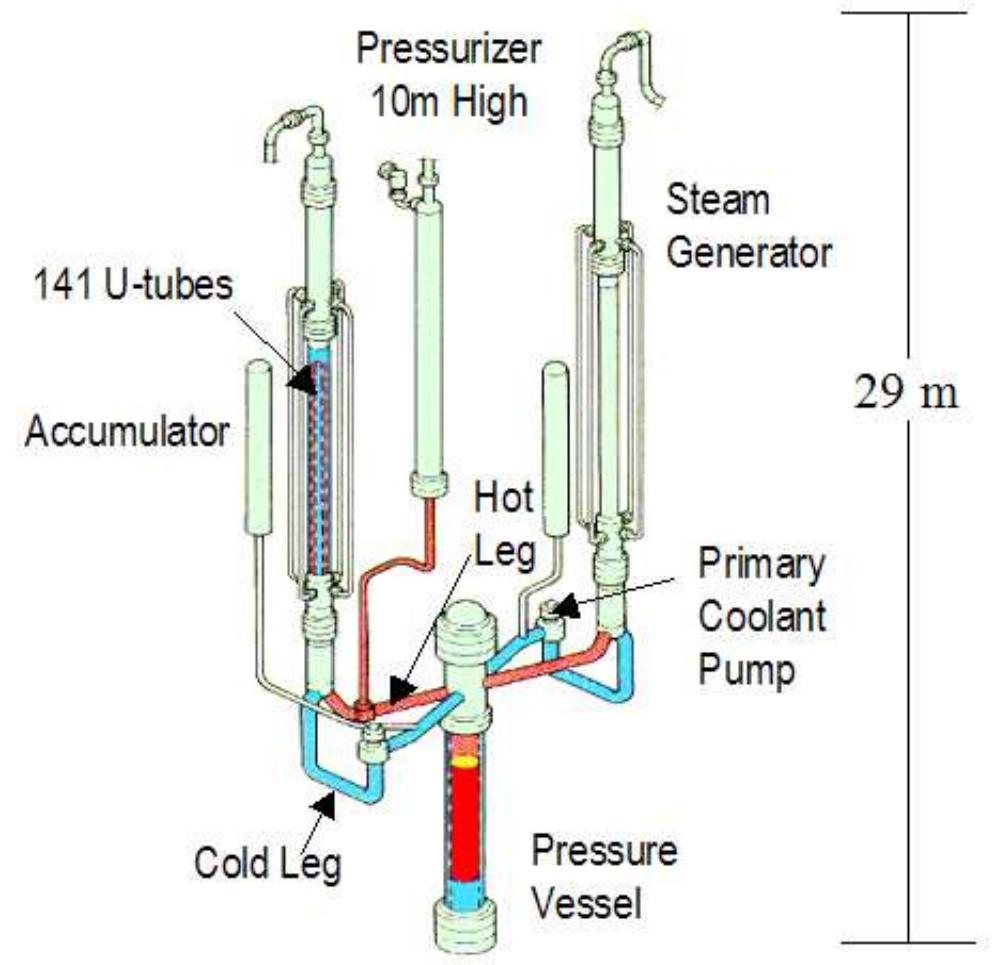

Fig. 1: Large Scale Test Facility

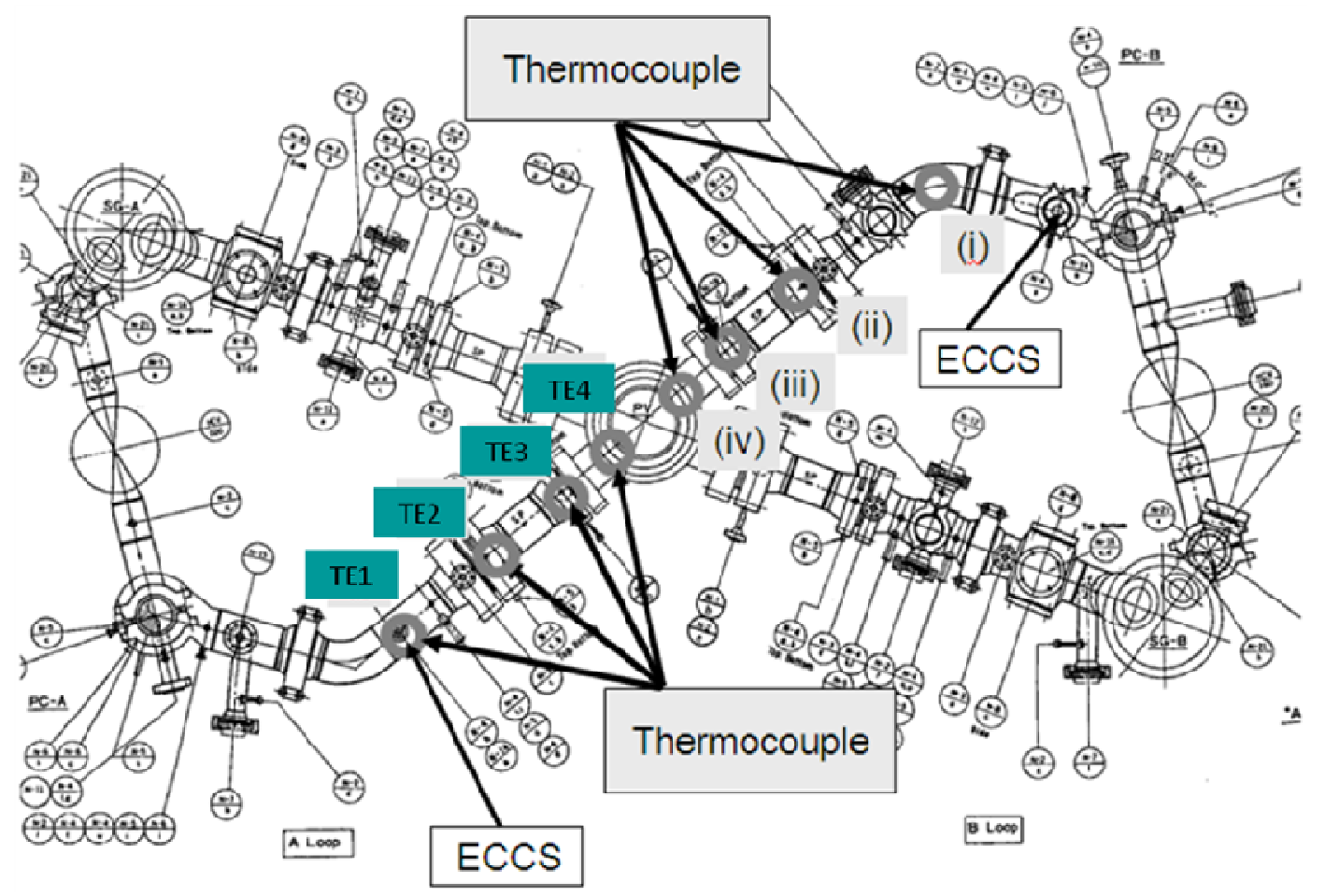

Fig. 2: Position of thermocouple rakes in Loops A and B 


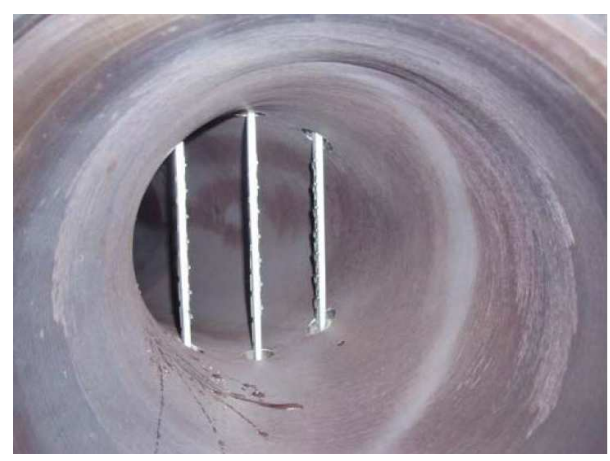

Fig. 3: Thermocouple installation in cold leg elbow section
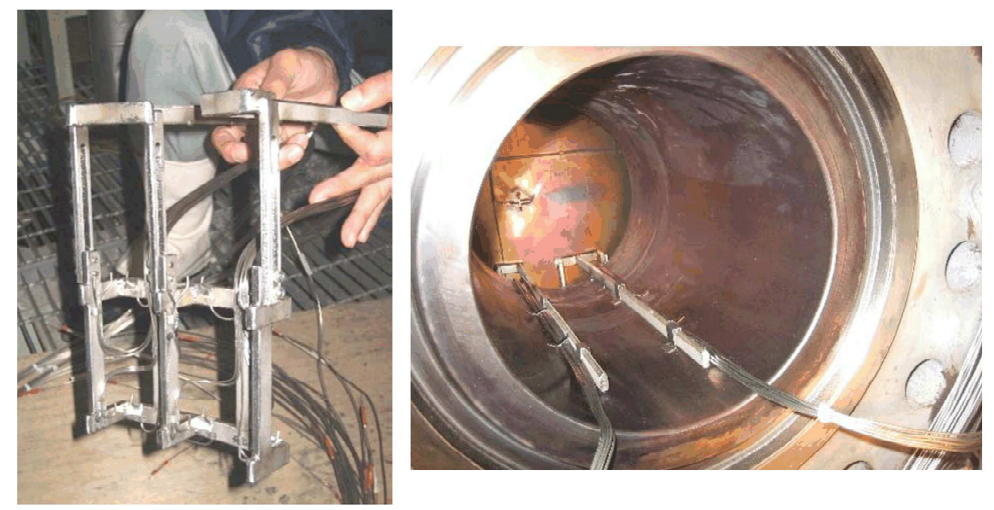

Fig. 4: Thermocouple installation in downcomer (left) and downcomer view from cold leg (right)

\section{CFD ANALYSIS}

The CFD analysis was performed for the first phase of Test 1-1 at $100 \%$ primary inventory and singlephase flow injection into Loop A. The ANSYS CFD software was used for the simulations.

\subsection{Geometry Generation}

A CAD model of the cold legs, the ECC lines, and the downcomer was generated on the basis of drawings (JAERI, 2003). The complete length of the downcomer was modelled to avoid feedback of boundary conditions on the flow in the section of interest. Following recommendations of Farkas et al. (2008), a rounded transition with a radius of $19 \mathrm{~mm}$ was used to connect the cold legs and the downcomer. The boundary conditions were specified at the cold legs directly after the primary coolant pumps, at the ECC lines, and at the lower part of the downcomer as shown in Fig. 5. 

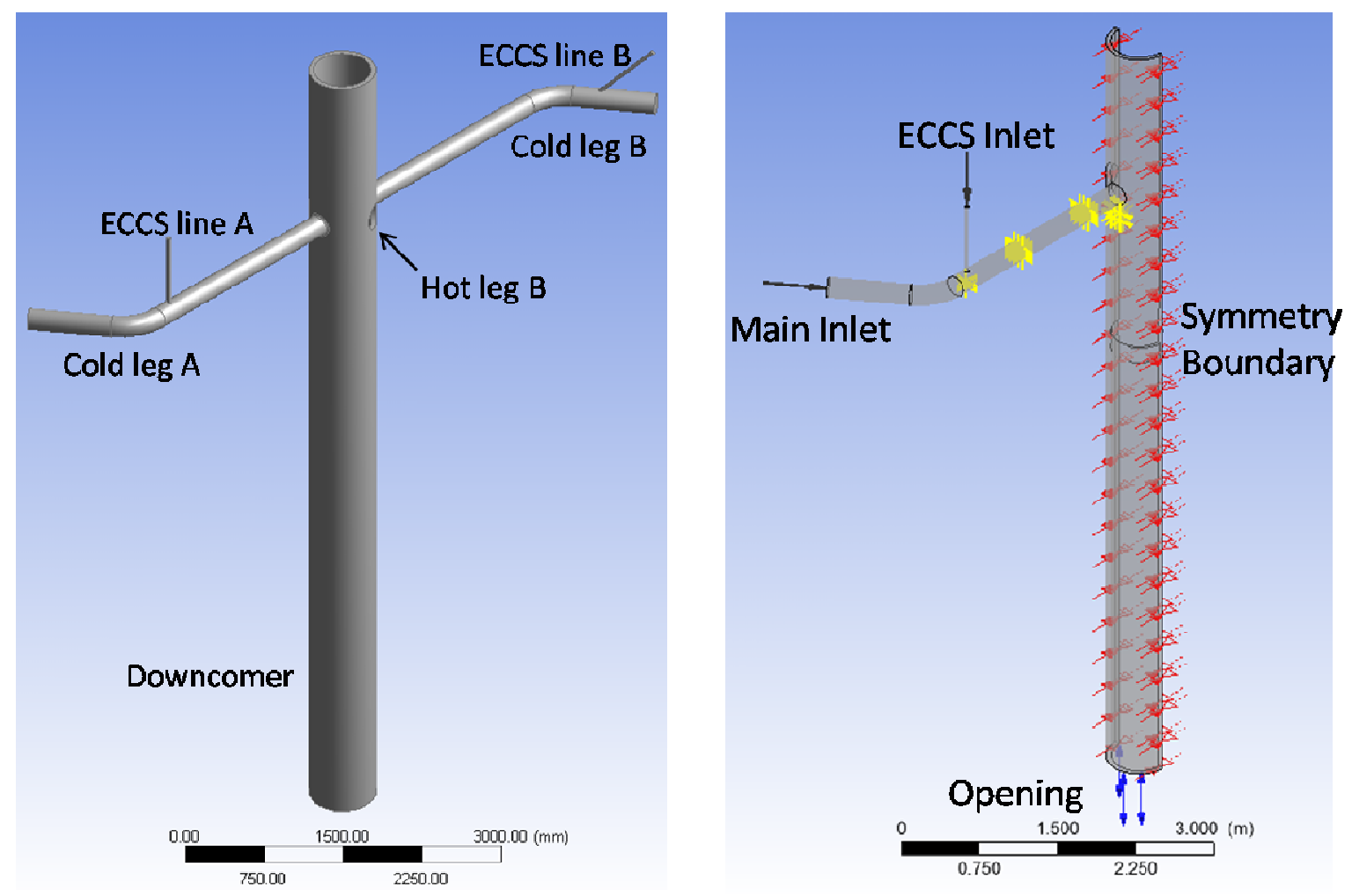

Fig. 5: Geometry model and boundary condition planes

\subsection{Grid Generation}

Following the OECD/NEA Best Practice Guidelines (Mahaffy et al., 2007), three hexahedral grids with increasing resolution were generated to perform sensitivity studies. In the first refinement step the number of elements was mainly increased in the cold leg. In the second step, the grid was additionally refined around the ECC nozzle. All generated grids were scalable with a minimum grid angle of $32^{\circ}$. Only $1 \%$ of the control volumes have grid angles with less than $45^{\circ}$. The resulting grids are shown in Fig. 6 for a cross section close to the inlet of the main pipe. The coarse grid has 1.0 million elements, the medium grid has 5.2 million elements, and the fine grid has 8.0 million elements for the complete geometry model.
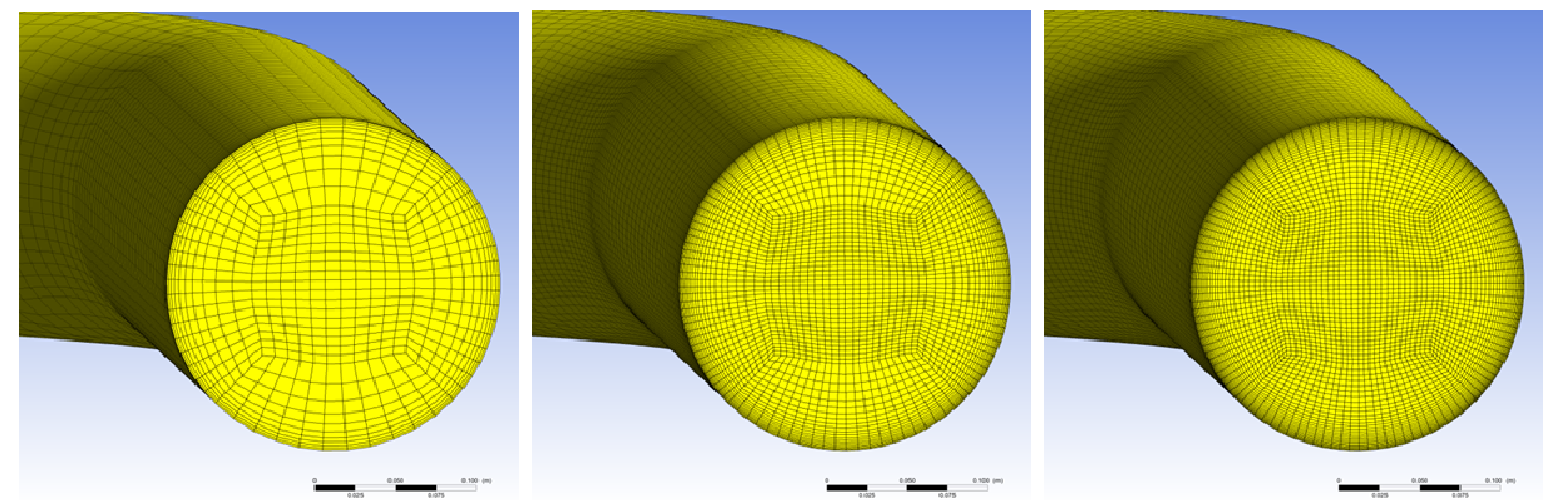

Fig. 6: Computational grids close to inlet of main pipe 


\subsection{Mathematical Models}

In the ROSA V Test 1-1 steady-state natural circulation is established in the primary loop and the main coolant pumps are stopped before ECC injection is started. The temperature difference between the hot water in the primary system and the cold ECC water is more than $200 \mathrm{~K}$ resulting in density differences of about $20 \%$. As a consequence, natural circulation is modelled in the CFD calculation by including buoyancy terms in the momentum equations, and in the production terms of the turbulence model equations. The buoyancy terms are driven by the density difference between the hot and cold water. Water density is calculated as a function of temperature on the basis of IAPWS-IF97 tables (Wagner and Kruse, 1998). The dynamic viscosity and conductivity of the fluid are also derived from the IAPWS-IF97 tables.

Two turbulence models were used in the simulation, the Shear Stress Transport (SST) model of Menter (1994) and a modification of the Reynolds stress model proposed by Launder et al. (1975). The modification of the Reynolds stress model concerns the length-scale equation. In contrast to the original proposal by Launder et al. (1995), a version using the turbulent vorticity as length-scale equation was used in this study. Both models were combined with 'automatic' wall functions in which the near-wall fluxes are derived from either linear or logarithmic wall laws, depending on the position of the wall-adjacent grid point.

\subsection{Initial and Boundary Conditions}

The transient calculations were started from a steady-state solution of the natural circulation. At the pump positions, and at the ECC injection nozzle A, the measured mass flow rates and temperatures were used as inlet boundary conditions. At the pump position, the inlet temperature was set to a constant value of 553.8 $\mathrm{K}$. The measured temperature at the ECC nozzle varied from $500 \mathrm{~K}$ to $300 \mathrm{~K}$ within $60 \mathrm{~s}$.

At the outlet, which was positioned at the lower part of the downcomer, a pressure boundary condition was prescribed. At the walls no-slip boundary conditions for smooth surfaces were used. The energy transfer between the fluid and the downcomer walls was set to zero (adiabatic walls). This was the best approximation of the experimental conditions, given that no temperature or heat flux measurements were available. The approximation of adiabatic walls has also been used by other authors, e.g. Farkas and Toth (2008).

\subsection{Numerical and Model Errors}

A $180^{\circ}$-degree half model was used for the investigations of numerical and model errors in order to economize on computing resources. As a first step, iteration errors were checked on each grid at steadystate conditions. The purpose of this study was to determine a convergence criterion of the iterative CFD solver such that iteration errors became insignificant. Fig. 7 shows temperatures at the measurement positions as a function of the convergence criterion. The temperatures did not change any more when the convergence criterion was reduced below $1 \times 10^{-4}$. As a consequence, a convergence criterion of $1 \times 10^{-4}$ was set in the calculations.

Discretization errors in time and space were also checked on each grid. The influence of the spatial numerical grid was investigated by comparing temperature transients on the three grids at the measurement position TE 1215 . As ROSA V data are proprietary, Fig. 8 shows only temperature differences instead of absolute temperature values. Large differences are observed between the coarse and medium grids. On the other hand, temperature differences on the medium and fine grids amount to less than $1 \mathrm{~K}$ in the final part of the transient. This is less than the thermocouple uncertainty of $\pm 2.75 \mathrm{~K}$. As a consequence, the medium-sized grid was chosen for the production calculations. It should be added that further grid refinement studies would be required to accurately quantify the solution errors as described by Mahaffy (2007). The comparison of calculations with first-order upwind and second-order high-resolution discretisation schemes are shown in Fig. 9. As expected, the second-order scheme predicts more oscillatory flow behaviour. The absolute temperature differences between the two schemes were, however, not larger than $2 \mathrm{~K}$. 
In order to save CPU time and to optimize the time step size, adaptive time stepping was used in combination with an implicit first-order time discretisation scheme. The influence of the initial time step of the adaptive scheme is shown in Fig. 10. The time step varied during the transient calculation between 0.01 and $1.0 \mathrm{~s}$ to achieve a convergence criterion of $1 \times 10^{-4}$ for the normalized residuals in all time steps. On average 17 iterations per time step were necessary for convergence for the calculations starting with 0.01 $\mathrm{s}$. The average influence of the time step size is around $8 \mathrm{~K}$ between 20 and $25 \mathrm{~s}$, and amounts to around 4 $\mathrm{K}$ between 25 and $35 \mathrm{~s}$. This corresponds to less than $2 \%$ error considering absolute temperature values. Spatial and temporal grid convergence of the solutions would require further refinement of time steps and grid widths.

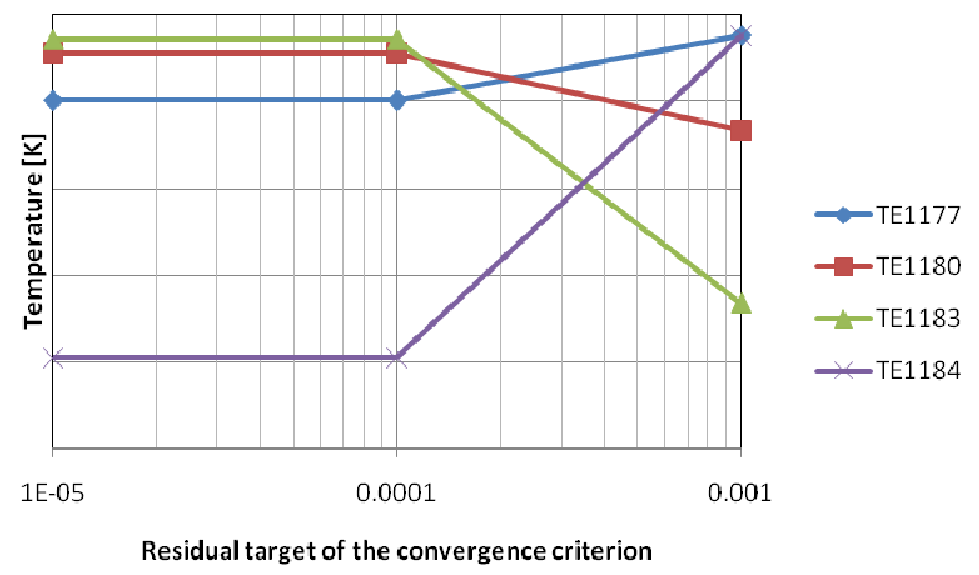

Fig. 7: Temperature as function of convergence criterion

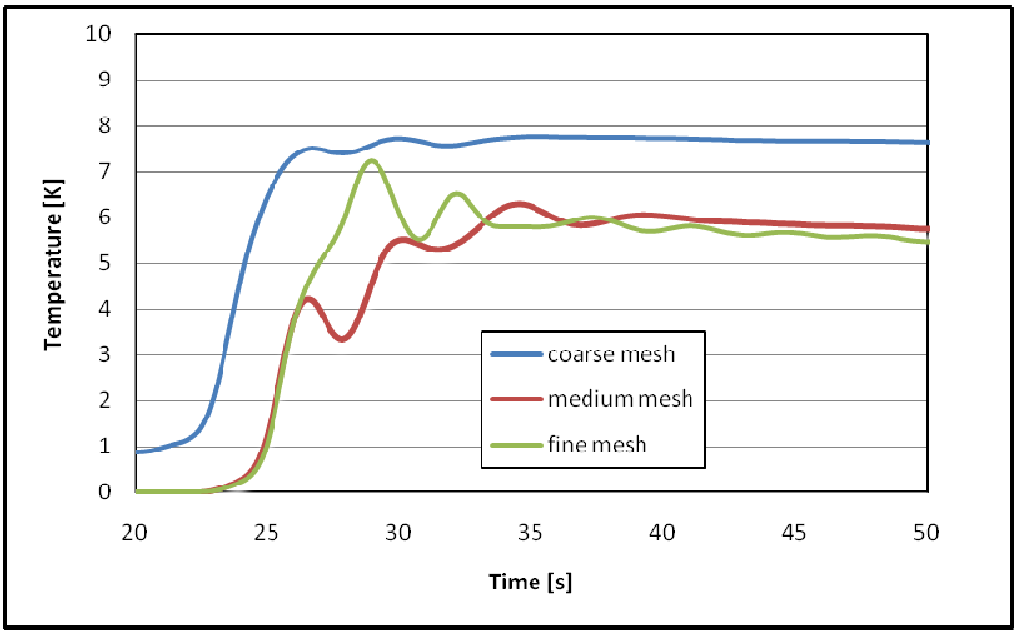

Fig. 8: Influence of spatial discretisation (TE1215) 


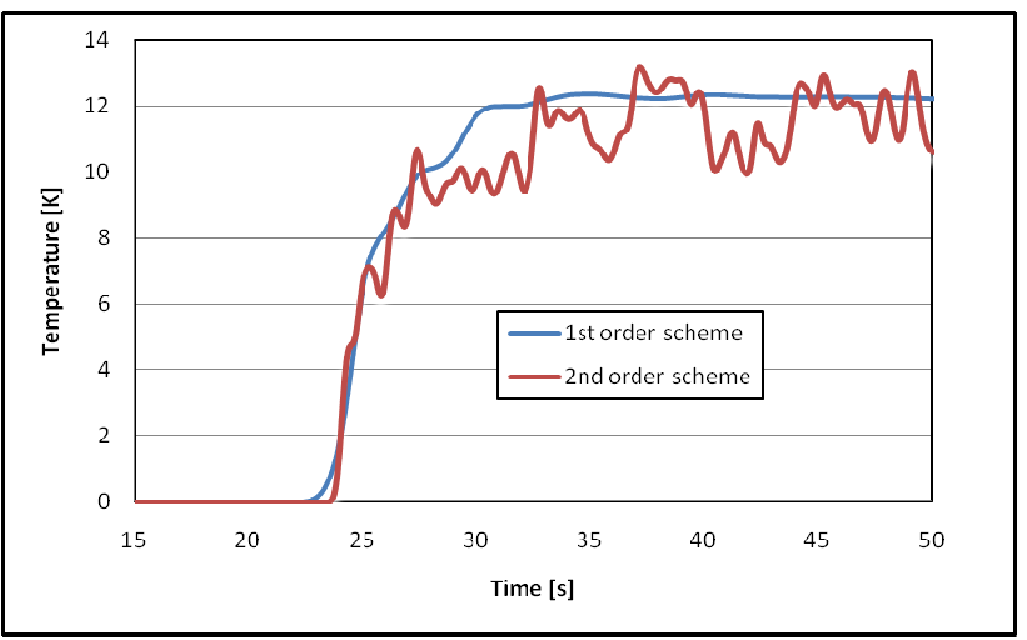

Fig. 9: Influence of discretisation scheme (TE1205)

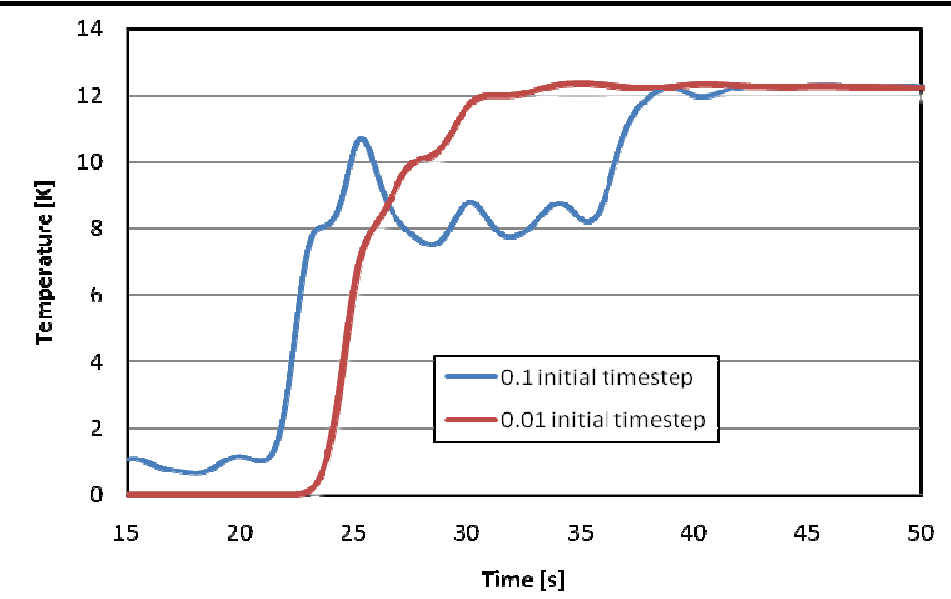

Fig. 10: Influence of initial time step size (TE1205)

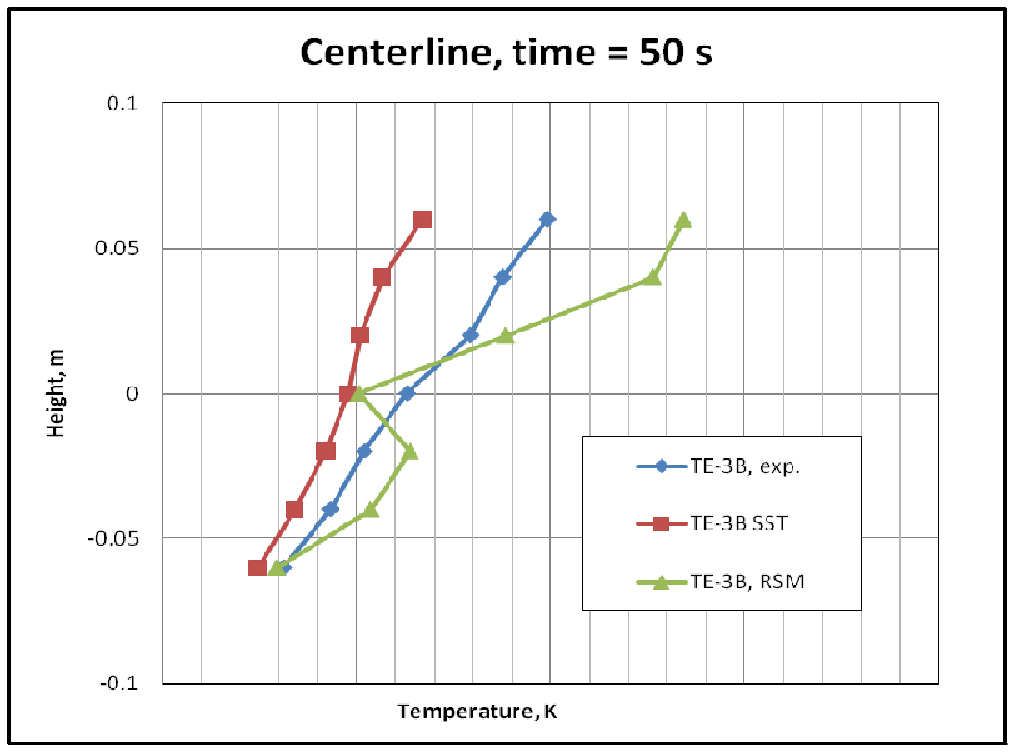

Fig. 11: Influence of turbulence model

Page 8 of 12 
Finally, the influence of the physical models was investigated. To this end, the SST model, and the Reynolds stress model (RSM) were compared. Fig. 11 shows as an example that there is no significant improvement from using the more complex RSM model, which took 1.4 times the calculation time of the SST model. Therefore, all further calculations were performed with the SST model.

\section{RESULTS AND COMPARISON TO DATA}

A $360^{\circ}$ geometry model was used for the comparison of simulations and data. As the application of the Best Practice Guidelines in section 3.5 had shown that the medium-sized grid produced the best results in terms of accuracy and calculation time a corresponding $360^{\circ}$ grid was generated for the production runs. This grid had 5.2 million elements. The calculations were performed with the ANSYS CFX software (Version 12) on a Sun Galaxy with 16 processors at $2.6 \mathrm{GHz}$. The final calculations of $115 \mathrm{~s}$ transient time took approximately 2 days calculation time.

An impression of the temperature distribution in Cold Leg A, and in the downcomer during ECC injection is shown in Fig. 12. The cold water in the cold leg shows stratification, and an oscillatory motion. The cold ECC water plume is transported downstream by the main flow and touches the bottom wall of the cold leg downstream of the injection nozzle. At this point, stratification is strongest. Downstream, the cold water plume is sloshing from side to side in the horizontal pipe and hot and cold water are mixed. As observed in the experiment, the cold water plume is entering the downcomer attached to the outer vessel wall.

A quantitative comparison of the temperature distribution in the downcomer is shown in Fig. 13. Close to the vessel wall (TE1225) experiment and calculation are in good agreement. At the barrel wall (TE1227) the calculated temperatures are higher, while the data indicate stronger mixing of the cold water plume with the surrounding hot water. However, the experimental flow may be influenced by the relative large fixtures of the thermocouples which could not be included in the simulations because no geometry information was available.
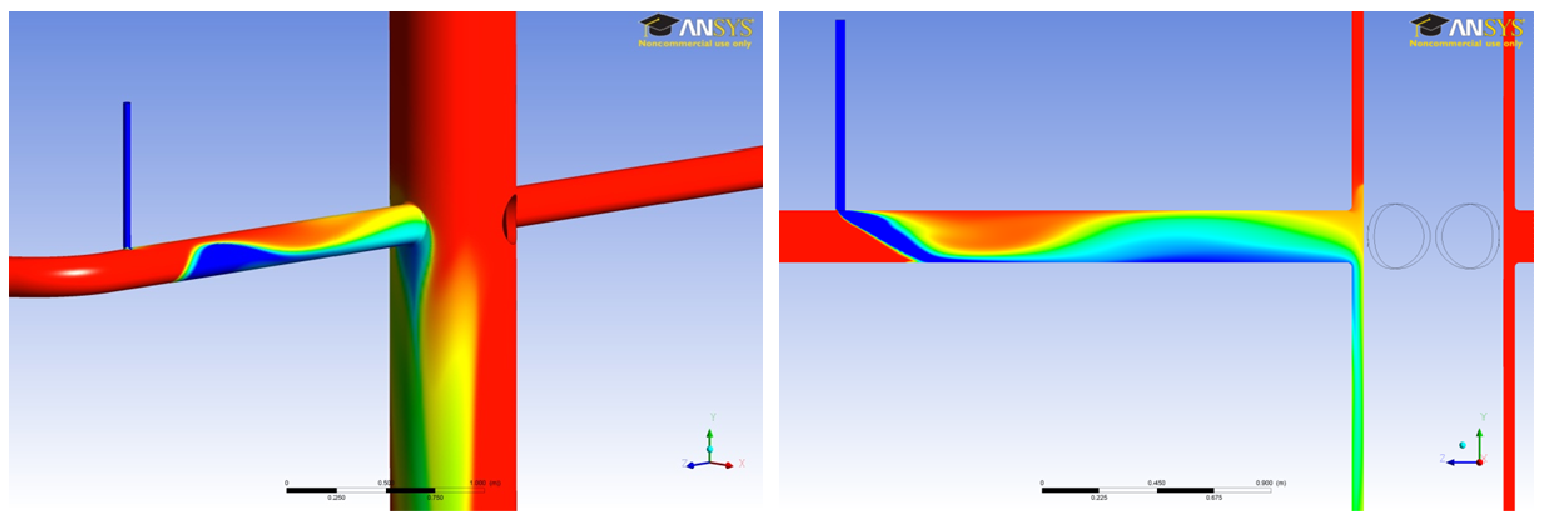

Fig. 12: Temperature stratification in Cold Leg A @ $80 \mathrm{~s}$ 


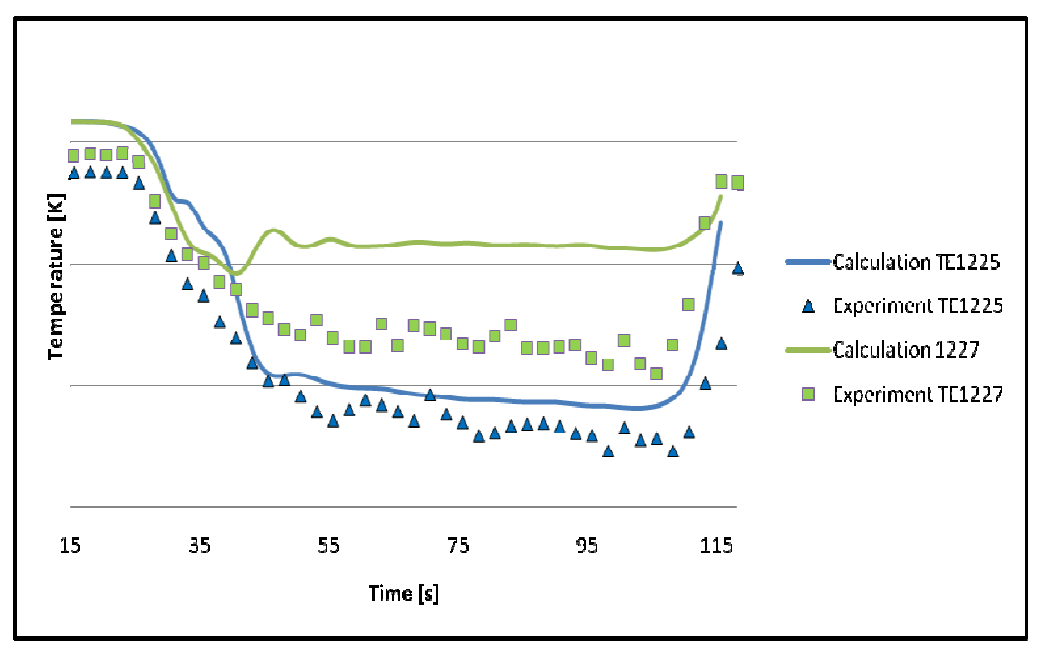

Fig. 13: Comparison of simulation and data in the downcomer (TE1225, TE1227)

Fig. 14 and Fig. 15 show the comparison to data at the central thermocouple position of rakes TE2 and TE3 in the cold leg, respectively. The red and black dashed lines show the measurement uncertainty of the thermocouples. At position TE2 (Fig. 14), which is close to the inlet nozzle, the calculation underpredicts mixing above the centreline of the horizontal pipe. The temperature in the upper part of the cold leg is therefore higher than in the experiment. At position TE3 (Fig. 15), the simulation results are in good agreement with data.

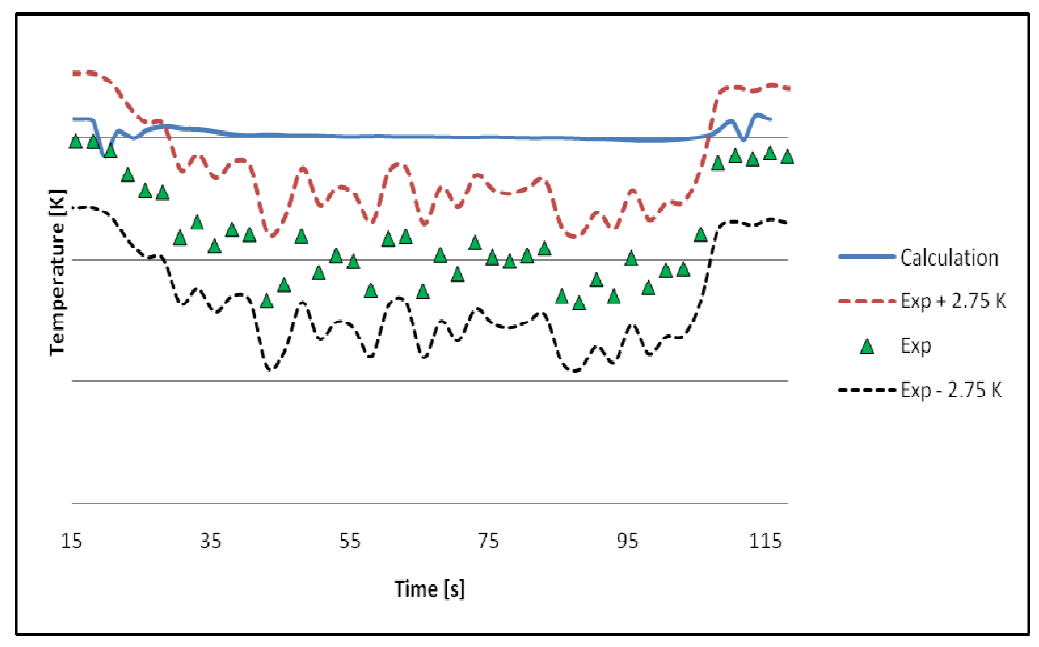

Fig. 14: Comparison of simulation and data in Cold Leg A (TE1187) 


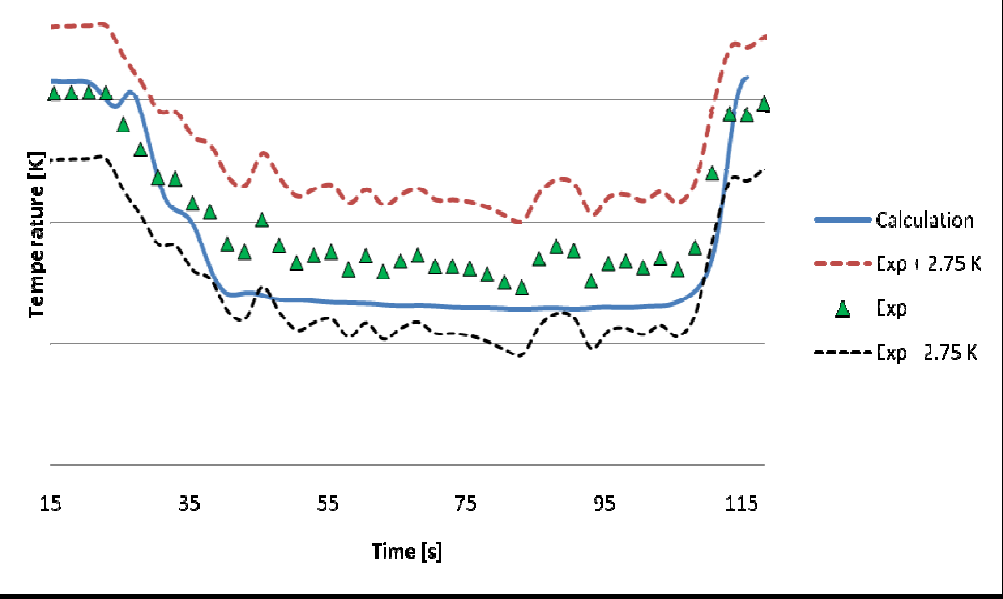

Fig. 15: Comparison of simulations and data in Cold Leg A (TE1208)

\section{CONCLUSIONS}

A numerical analysis of the ROSA V Test 1-1 was performed with the ANSYS CFD software. The ROSA V experiment investigates thermal stratification at single-phase natural circulation conditions in the cold leg of a PWR. The numerical results were compared with temperature measurements at three rake positions in Cold Leg A, and at one position in the downcomer below the cold leg.

The numerical grid generation and error analysis was performed according to the OECD Best Practice Guidelines. After reduction of numerical errors, model errors were investigated by comparing different turbulence models. The final comparison with data showed good agreement. It leads to the following conclusions:

- Close to the ECC injection nozzle, turbulent mixing induced at the shear layer between the cold ECC-water and the hot water in the cold leg is under-predicted. In order to improve agreement between predictions and data use of a scale-resolving turbulence model may be necessary.

- Temperature stratification along the cold leg and in the downcomer is in good agreement with data which indicates that the buoyancy-driven stratification is accurately simulated.

- The attachment of the cold water plume to the outer wall of the pressure vessel in the downcomer is captured by the CFD simulation.

The calculations indicate the predominance of buoyancy effects caused by density difference between cold and hot water in the cold leg. The temperature measurements indicate that mixing effects are more dominant. However, the mixing effect of the thermocouple rakes and the video probe installed in the flow field were not investigated. Further studies will concentrate on an increased resolution of the area around the ECC nozzle and the application of scale-resolving turbulence models.

\section{ACKNOWLEDGMENTS}

This work has been funded by the European Commission in the frame of the NURESIP-project. We would like to acknowledge the support of the German Federal Ministry of Economics and Technology under Contract RS 1188. We would like to thank Professor Rafael Macian of the Technische Universität München for his support of the diploma thesis of Johannes Weis and we are grateful to Tanja Farkas and Ivan Toth of KFKI, Budapest, for helpful discussions during the course of this work. 


\section{REFERENCES}

T. Farkas, I. Tóth, "FLUENT Analysis of a ROSA Cold Leg Stratification Test”, Internal Report, Hungarian Academy of Sciences, KFKI, Atomic Energy Research Institute, Budapest (2008)

T. Höhne, S. Kliem, M. Scheuerer, "Experimental and Numerical Modelling of a Buoyancy-Driven Flow in a Reactor Pressure Vessel", The 11th Int. Topical Meeting on Nuclear Reactor Thermal-Hydraulics (NURETH-11), Avignon, France, 2-6 October, Paper 480, (2005).

JAERI, "ROSA V Large Scale Test Facility (LSTF) System Description for the Third and Fourth Simulated Fuel Assemblies", Tokai Research Establishment, Japan Atomic Energy Research Institute (2003)

JAERI, "Final Data Report of OECD/NEA ROSA Project Test 1-1", Thermohydraulic Safety Research Group, Nuclear Safety Research Centre, Japan Atomic Energy Agency (2008)

B. E. Launder, G. Reece, W. Rodi, "Progress in the development of a Reynolds stress turbulence closure", J. Fluid Mech. 68:537-66 (1975)

J. Mahaffy, "Best Practice Guidelines for the Use of CFD in Nuclear Reactor Safety Applications", NES/CSNI/R(2007)5 (2007)

F. R. Menter, "Two-Equation Eddy-Viscosity Turbulence Models for Engineering Applications", AIAAJournal, Vol. 32, pp. $269-289$ (1994)

M. Scheuerer, “Selection of PTS-Relevant Test Cases,” EVOL-ECORA-D05a (2002)

W. Wagner, and A. Kruse, The Industrial Standard IAPWS-IF97: Properties of Water and Steam, Springer-Verlag, Berlin (1998)

S. M. Willemsen, M. J. Komen, "Assessment of RANS CFD modelling for Pressurized Thermal Shock Analysis", $11^{\text {th }}$ Int. Topical Meeting on Nuclear Reactor Thermal-Hydraulics (NURETH-11), Avignon, France, Paper 121, (2005) 